\title{
A REMARK ON A THEOREM OF A. WEIL
}

\section{MORIKUNI GOTO ${ }^{1}$}

1. The purpose of this paper is to prove the following theorem.

THEOREM. Let $G$ be a connected semisimple Lie group without compact components. Let $H$ be a subgroup of $G$ such that there exists a compact subset $K$ of $G$ with $G=H K$. Let $\sigma$ be a continuous automorphism of $G$ which reduces to the identity on $H$. Then $\sigma$ is the identity automorphism of $G$.

This is a generalization of a theorem of A. Weil in [4], and is related to a theorem of $\mathrm{A}$. Borel in [1]. The author obtained the theorem by globalizing the infinitesimal method of Weil in [4].

2. Let $G$ be a connected semisimple Lie group. Let $A(G)$ be the group of all continuous automorphisms of $G$. $A(G)$ is a Lie group with respect to the compact-open topology. Let $I(G)$ be the subgroup of $A(G)$ composed of all inner automorphisms. Then $I(G)$ is a closed normal subgroup of finite index in $A(G)$. For $g$ in $G$ we define $\operatorname{Ad}(g)$ by $\operatorname{Ad}(g) h=g h g^{-1}(h \in G)$. Then $G \ni g \mapsto \rightarrow \operatorname{Ad}(g) \in I(G)$ gives a continuous homomorphism, whose kernel coincides with the center of $G$. Let $(B)$ be the Lie algebra of $G$, and let $\oiint_{1}, B_{2}, \cdots, B_{k}$ be the simple factors of $(B): B=B_{1} \oplus \mathfrak{S}_{2} \oplus \cdots \oplus \mathbb{S}_{k}$ (direct sum of ideals). Let $I(\mathbb{S}), I\left(\mathbb{S}_{1}\right), \cdots$, and $I\left(\mathbb{S}_{k}\right)$ be the adjoint groups of $\mathfrak{S}, \mathfrak{H}_{1}, \cdots$, and $\mathfrak{S H}_{k}$ respectively. $I(\mathfrak{S})$ can be naturally identified with $I(G)$, and we have

$$
I(G)=I\left(\mathfrak{S}^{\ddagger}\right)=I\left(\mathbb{G}_{1}\right) \times I\left(\mathfrak{G}_{2}\right) \times \cdots \times I\left(\mathfrak{G}_{k}\right)
$$

(direct product of closed normal subgroups). We denote by $\epsilon$ the identity automorphism of $G$.

Lemma 1. Let $G$ be a connected semisimple Lie group. Let $N$ be a nontrivial, i.e., $N \neq\{\epsilon\}$, normal subgroup of $A(G)$. Then there exists an $i(i=1,2, \cdots$, or $k)$ with $N \supset I\left(\$_{i}\right)$.

Proof. First suppose that $N \cap I(\xi)=\{\epsilon\}$. Let $\sigma$ be in $N$. For $g$ in $G$ we have $\operatorname{Ad}(g) \sigma=\sigma \operatorname{Ad}(g)$, which implies that $\sigma\left(g^{-1}\right) g$ is in the center of $G$. On the other hand, $G \ni g l \rightarrow \sigma\left(g^{-1}\right) g$ gives a continuous map from the connected space $G$. Since the center of $G$ is discrete, we have

Received by the editors September 11, 1967.

${ }^{1}$ Research supported in part by NSF Grant GP 4503. 
$\sigma\left(g^{-1}\right) g=$ the unit element for all $g$ in $G$, i.e. $\sigma=\epsilon$. Hence $N=\{\epsilon\}$. This contradiction implies that $N \cap I(\mathfrak{B}) \neq\{\epsilon\}$.

Since each $I\left(\mathfrak{B}_{i}\right)(i=1,2, \cdots, k)$ has no proper normal subgroup, i.e. $I\left(\mathfrak{S}_{i}\right)$ is a simple group, see Goto [3], any nontrivial normal subgroup of $I(\mathbb{S})=I\left(\mathbb{S}_{1}\right) \times I\left(\mathbb{S}_{2}\right) \times \cdots \times I\left(\mathbb{S}_{k}\right)$ contains at least one of the $I\left(\mathbb{S}_{i}\right)$. Q.E.D.

3. Let $L$ be a topological group. For $a$ in $L$, we denote by $C(a)$ the conjugate class containing $a$. We define a subset $\mathbb{E}(L)$ of $L$ by the condition: $a \in \mathbb{C}(L)$ if and only if the closure of $C(a)$ is compact. Then the following lemma holds obviously.

LeMma 2. $\mathfrak{S}(L)$ is a normal subgroup of $L$.

Proposirion. Let $G$ be a connected semisimple Lie group without compact components. Then $\mathbb{E}(A(G))=\{\epsilon\}$.

Proof. If it is not true, then by Lemma 1 and Lemma $2 \mathfrak{C}(A(G))$ must contain some $I\left(\mathfrak{S}_{i}\right)$. Hence it suffices to prove that $I\left(\mathbb{S}_{i}\right)$ contains a closed subgroup $M$ with $\mathfrak{E}(M) \neq M$.

Since $B_{i}$ is a noncompact simple Lie algebra, there exists a subalgebra $\mathfrak{M}$ of $\mathfrak{B}_{i}$, which is isomorphic with $\operatorname{sl}(2, \boldsymbol{R})$, the Lie algebra of all real 2 by 2 matrices with trace 0 . (See Goto [2].) Since $I\left(\mathcal{G}_{i}\right)$ is a Lie group composed of linear transformations, the analytic subgroup $M$ of $I\left(G_{i}\right)$, corresponding to $M$, is closed and is isomorphic with $\mathrm{SL}(2, R)$, the real special linear group of two dimension, or with $I(\mathrm{SL}(2, R))$. Since the conjugate class containing

$$
\left(\begin{array}{ll}
1 & 1 \\
0 & 1
\end{array}\right)
$$

in $\operatorname{SL}(2, R)$ contains all

$$
\left(\begin{array}{ll}
1 & \alpha \\
0 & 1
\end{array}\right) \quad(\alpha>0)
$$

we have $\mathfrak{S}(M) \neq M$. Q.E.D.

4. Proof of Theorem. Let $\sigma$ be a continuous automorphism of G. Since $\sigma(h)=h$ implies that $\operatorname{Ad}(h) \sigma=\sigma \operatorname{Ad}(h)$, if $\sigma(h)=h$ for all $h$ in $H$, then

$$
\left\{\operatorname{Ad}(g) \sigma \operatorname{Ad}\left(g^{-1}\right) ; g \in G\right\}=\left\{\operatorname{Ad}(k) \sigma \operatorname{Ad}\left(k^{-1}\right) ; k \in K\right\}
$$

is compact. Since $A(G) / I(G)$ is finite, $C(\sigma)$ is compact in $A(G)$. Hence $\sigma \in \mathbb{S}(A(G))$, and by the Proposition $\sigma=\epsilon$. Q.E.D. 
In a recent conversation with $\mathrm{J}$. Tits, the author discovered that the main part of this paper is contained in J. Tits, Automorphismes a deplacement borné des groupes de Lie, Topology 3 (1964), 97-107.

\section{BIBLIOGRAPHY}

1. A. Borel, Density properties for certain subgroups of semi-simple groups without compact components, Ann. of Math. 72 (1960), 179-188.

2. M. Goto, Lattices of subalgebras of real Lie algebras, J. Algebra (to appear).

3. - On an arcwise connected subgroup of a Lie group, Proc. Amer. Math. Soc. 20 (1969), 157-162.

4. A. Weil, On discrete subgroups of Lie groups. II, Ann. of Math. 75 (1962), 578602 .

University of Pennsyluania 